\title{
Spin Dependent Interactions of Free Carriers and Manganese Ions in Nanostructures of Wide Band Gap II-Mn-VI Semiconductors - Mechanism of Lifetime Reduction
}

\author{
S. Yatsunenko ${ }^{a}$, A. Khachapuridze ${ }^{a}$, V.Yu. Ivanov ${ }^{a}$, \\ M. GODLEWSKI ${ }^{a, b}$, Le VAN KHOI ${ }^{a}$, Z. GOlaCKI ${ }^{a}$,

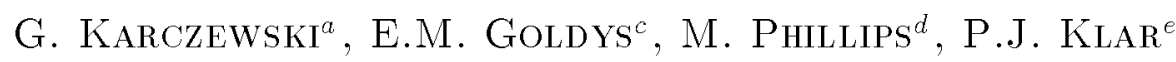 \\ AND W. HeIMBRodT ${ }^{e}$ \\ ${ }^{a}$ Institute of Physics, Polish Academy of Sciences \\ al. Lotnikow 32/46, 02-668 Warsaw, Poland \\ ${ }^{b}$ College of Science, Cardinal S. Wyszyński University, Warsaw, Poland \\ ${ }^{c}$ Division of Information and Communication Sciences \\ Macquarie Univ., Sydney, Australia \\ ${ }^{d}$ Microstructural Analysis Unit, UTS, Sydney, Australia \\ ${ }^{e}$ Dept. of Physics and Materials Sciences Center, Phillips-University of Marburg \\ Renthof 5, 35032 Marburg, Germany
}

Based on the results of optically detected magnetic resonance and time-resolved investigations we relate the observed lifetime shortening of intra-shell $\mathrm{Mn}^{2+}$ emission to spin dependent magnetic interactions between localized spins of $\mathrm{Mn}^{2+}$ ions and spins/magnetic moments of free carriers. We show that this mechanism is active in both bulk and in low dimensional structures, such as quantum wells, quantum dots, and nanostructures.

PACS numbers: 71.55.Gs, 76.30.Fc, 76.70.Hb, 78.55.Et

\section{Introduction}

In mid 80 s it was reported that manganese doped semiconductor nanostructures show high photoluminescence (PL) yield simultaneously with short PL decay 
times $[1,2]$. These properties made them suitable for a range of applications, such as fluorescent labels, phosphors, sensors, lasers and displays [3]. The most unusual feature of such structures was a rapid decrease in the lifetime of $\mathrm{Mn}^{2+}$ intra-shell emission $[1,2]$. The observed shortening of the PL decay time could not be related to a competition of nonradiative recombination, since shorter PL decay times were observed together with high quantum efficiency of the emission $[1,2,4-6] .{ }^{4} T_{1}$ to ${ }^{6} A_{1}$ transition is a parity and spin forbidden process and thus typically shows low recombination rate. PL decay times in the range of ms are commonly observed for Mn doped wide band gap II-VI compounds.

The reduction of the PL decay time was first observed for ZnS nanostructures doped with $\mathrm{Mn}[1,2,4]$. In this paper we report that $\mathrm{PL}$ shortening is observed not only for nanostructures, quantum well (QW), and quantum dot (QD) structures, but also for bulk samples. Moreover, a fast component of the PL decay is always observed together with a "normal" slow one. Thus, our observations question the model proposed by Bhargava [1, 2], who related PL enhancement to quantum confinement effects active in nanostructures. We propose that efficient spin flip interactions between $\mathrm{Mn}$ ions and free carriers are responsible for the observed effect.

\section{Experimental}

The experiments were performed on the range of $\mathrm{ZnMnS}$ (also CdMnS) nanostructures synthesized in the mesoporous MCM-41 $\mathrm{SiO}_{2}$ matrices [7-9]. Bulk $\mathrm{ZnMnS}$ crystals with about $1 \% \mathrm{Mn}$ fraction grown by the chemical transport method were studied for the comparison. Test measurements were also performed on self-organized CdMnTe QD system grown by molecular beam epitaxy on ZnTe, for CdMnTe/CdMgTe QWs, and also for ZnMnSe and ZnMnTe bulk samples.

PL and PL kinetics experiments were performed using either a second or third harmonics of YAG:Nd pulsed laser for the PL excitation and photon counting system for the detection. The scanning electron microscopy (SEM) images were taken with LEO computer controlled field emission scanning electron microscope.

PL and PL kinetics investigations were supplemented with optically detected magnetic resonance (ODMR) and electron spin resonance (ESR) investigations. Most of the ESR studies were done with an X-band Bruker spectrometer. ODMR (and also a part of the ESR) investigations were performed on a Q-band ( $36 \mathrm{GHz}$ ) system developed by the authors, with a microwave cavity mounted in a split-coil magnet of the Oxford Instruments. PL changes at magnetic resonance conditions were measured in phase with on-off modulated microwave power. Spectral response of the magnetic resonances, the so-called ODMR-PL spectrum, was measured by scanning with monochromator spectral response of the resonance, once keeping magnetic field, microwave power, and modulation frequency of microwaves optimized for the detection of the ODMR signals. 


\section{Results and discussion}

In Fig. 1 we show SEM image taken for $\mathrm{ZnMnS}$ nanostructure with $30 \%$ Mn fraction. The small and sticked together nano objects of 10-40 nm size are observed, which are forming larger grains of a micrometer size. Such size of nano grains indicates that eventual quantum confinement effects should be weak, if any. Similar microstructure are observed for all nanostructures of $\mathrm{ZnMnS}$ and $\mathrm{CdMnS}$ studied.

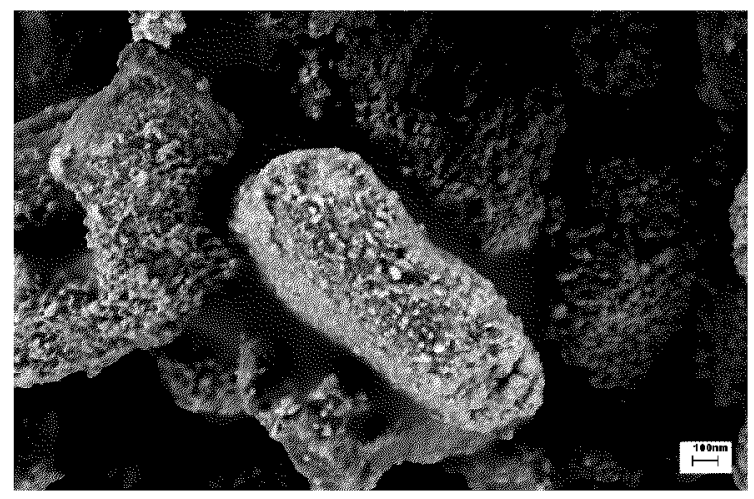

Fig. 1. Scanning electron microscopy image of $\mathrm{ZnMnS}$ nanocrystals taken at $1.4 \mathrm{kV}$ accelerating voltage from $1.96 \times 3 \mu \mathrm{m}$ area.

In Fig. 2 we show PL spectrum of ZnMnS nanostructures measured at room temperature under the above band gap excitation. An asymmetric PL emission is observed in the spectral range of $1.6-2.2 \mathrm{eV}$. The spectral position is similar to the one for bulk $\mathrm{ZnMnS}$, as shown for the comparison. However, PL of nanostructures shows a pronounced low energy wing, which extends down to $1.6 \mathrm{eV}$. This low energy wing can be due to some underlying PL of a different origin.

In Fig. 2 we show the so-called ODMR-PL spectrum, i.e., spectral response of the $\mathrm{Mn}^{2+}$ magnetic resonance. $\mathrm{Mn}^{2+}$ magnetic resonance is observed from all spectral region of PL in bulk ZnMnS. Thus, based on the latter observation, and the ESR study showing strong $\mathrm{Mn}^{2+}$ magnetic resonance, we attribute the dominant part of the observed PL to ${ }^{4} T_{1}$ to ${ }^{6} A_{1}$ intra-shell emission of $\mathrm{Mn}^{2+}$ ions. The comparison of the spectra indicates that PL of $30 \% \mathrm{ZnMnS}$ nanostructures is fairly similar to the one of $1 \%$ (Mn fraction) bulk $\mathrm{ZnMnS}$. We do not observe any spectral shift of the PL or intensity redistribution. In the ODMR-PL the PL emission is enhanced at high-energy wing, due to the contribution of Mn pairs, associates etc. Consequently, we expected similar enhancement/shift in nanostructures, since most of Mn ions should be paired. This is not seen. Also in the ESR we observed intensive magnetic resonance of isolated Mn ions. These observations suggest that either Mn fraction in nanostructures is well below the intended one, or that some of Mn ions, when close to a surface of nanostructures, do not find 


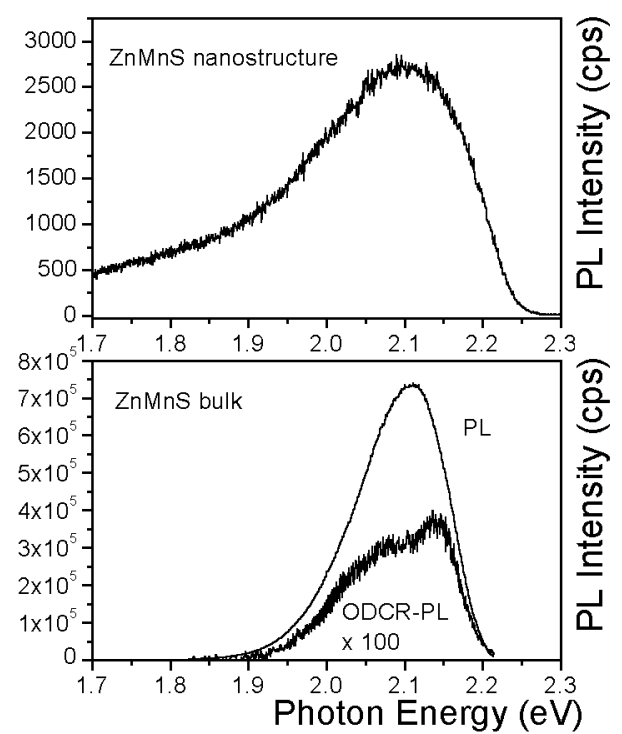

Fig. 2. Room temperature photoluminescence (PL) spectrum of $\mathrm{ZnMnS}$ ( $30 \%$ of $\mathrm{Mn}$ ) nanocrystals, as compared to the low temperature PL and ODMR-PL spectra taken for bulk ZnMnS ( $1 \%$ of Mn).

nearby neighbours, which would explain the observed properties of the PL and ESR spectra.

Once we identified intra-shell emission of $\mathrm{Mn}^{2+}$, we measured its PL decay kinetics. Room temperature data are shown in Fig. 3 together with those measured for bulk ZnMnS under the above band gap excitation. For both systems PL kinetics shows a very pronounced fast component of the decay. This fast decay component, observed together with a "normal" slow decay component of ms range, we attribute to a competition of two channels of a radiative decay. We thus reject the idea that the observation of the fast component of the PL decay reflects competition of some channel of a nonradiative decay. As already mentioned fast components of the PL decay were reported for nanostructures showing simultaneously a high quantum efficiency of the PL. A similar situation is expected in the present case. We observed a relatively bright PL for nanostructures embedded into mesoporous $\mathrm{SiO}_{2}$ matrices. Such embedding likely passivates possible surface states, which are active in nonradiative transitions.

Bol and Meijerink [4] related the fast PL decay component in nanostructures of $\mathrm{ZnMnS}$ to an underlying PL of a different origin. We tested this idea by comparing PL spectra measured for different nanostructures and bulk samples. In the case of bulk ZnMnS we found that the green PL of ZnS in fact underlies the Mn intra-shell emission. Moreover, the time-resolved study confirmed that the green PL band in ZnMnS decays very fast, which, based on the ODMR study, we explain 
by efficient spin dependent energy transfer from the donor-acceptor pairs, active in the green band PL, to Mn ions.

For our ZnMnS nanostructures such explanation of the fast PL decay component is also possible. Even though we have not observed an underlying green PL band in this system, another PL (red PL) overlaps a low energy wing of the $\mathrm{Mn}^{2+}$ emission. We performed PL and PL kinetics measurements for several other samples to be positively convinced that fast decay component of the $\mathrm{Mn}^{2+} \mathrm{PL}$ is not an artefact. Nanostructures of CdMnS, QD and QW structures of CdMnTe, and bulk samples of ZnMnSe, ZnMnTe, and CdMnTe were studied. In each case a fast component of the PL decay was observed together with a slow one, which convinced us that this decay component must be due to a common recombination mechanism, which is present in all samples doped with $\mathrm{Mn}$ ions. We also notice that the effect is not necessarily more pronounced in low dimensional structures. The latter we conclude from e.g. comparison PL decays shown in Fig. 3.

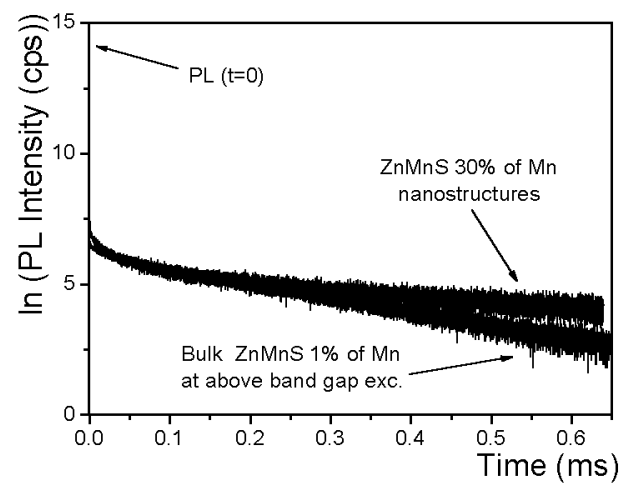

Fig. 3. PL kinetic of $\mathrm{Mn}^{2+}$ intra-shell emission in nanocrystals of $\mathrm{ZnMnS}$ ( $\mathrm{Mn}$ fraction of 0.3 ), as compared to the PL decay observed for the bulk $\mathrm{ZnMnS}$ with $1 \% \mathrm{Mn}$ fraction. A slow component of the PL decay follows extremely sharp initial PL decay, seen as a nearly vertical line.

Our recent investigations of PL kinetics in CdMnTe quantum dots indicate that rise and decrease (fast component) of the $\mathrm{Mn}^{2+} \mathrm{PL}$ correlates with the time of trapping of light-generated free carriers into excitons [6]. Moreover, our recent ODMR studies of $\mathrm{CdMnTe} / \mathrm{CdMgTe} \mathrm{QW}$ system [5] indicate that interaction of free carriers with localized spins of $\mathrm{Mn}$ ions can be efficient and can result in a significant modification of the PL spectrum. These observations are in line with the present ones. For example, we notice that PL decay of Mn intra-shell emission shortens if we use the above band gap excitation. The fast component of the PL decay is less pronounced under intra-shell excitation.

All these observations allow us to propose that very efficient spin flip scattering of free carriers (mainly of free holes [10]) results in relaxation of spin selection 
rules for the intra-shell PL of $\mathrm{Mn}^{2+}$ ions. Then, the mechanism should be common and should be observed both in nanostructures and bulk samples, as we demonstrate in the present study.

\section{Conclusions}

Concluding, a fast component of the $\mathrm{Mn}^{2}+\mathrm{PL}$ decay is not related to quantum confinement effects, as suggested before, and is present in both low dimensional structures and in bulk samples. We relate the observation of this component of the PL decay to efficient spin flip interactions between localized spins of $\mathrm{Mn}$ ions and spins of free carriers. These interactions can relax spin selection rules for $\mathrm{Mn}^{2+}$ intra-shell transitions.

\section{Acknowledgment}

The work was partly supported by the grant numbers 5 P03B 00720 and 7 T08A 00620 (Z. Gołacki) of the State Committee for Scientific Research for the years $2001-2003$.

\section{References}

[1] R.N. Bhargava, J. Lumin. 70, 85 (1996).

[2] R.N. Bhargava, D. Gallagher, Phys. Rev. Lett. 72, 416 (1994).

[3] T. Trindade, P. O'Brien, N. Pickett, Chem. Matter 13, 3843 (2001).

[4] A.A. Bol, A. Meijerink, Phys. Rev. B 58, R15997 (1998).

[5] M. Godlewski, V.Yu. Ivanov, A. Khachapuridze, S. Yatsunenko, Phys. Status Solidi B 229, 533 (2002)

[6] M. Godlewski, V.Yu. Ivanov, P.J. Bergman, B. Monemar, Z. Gołacki, G. Karczewski, J. Alloys Comp. 341, 8 (2002).

[7] L. Chen, P.J. Klar, W. Heimbrodt, F. Brieler, M. Fröba, Appl. Phys. Lett. 76, $3531(2000)$.

[8] L. Chen, P.J. Klar, W. Heimbrodt, F. Brieler, M. Fröba, H.-A. Krug von Nidda, A. Loidl, Physica E 10, 368 (2001).

[9] L. Chen, H. Falk, P.J. Klar, W. Heimbrodt, F. Brieler, M. Fröba, H.-A. Krug von Nidda, A. Loidl, Z. Chen, Y. Oka, Phys. Status Solidi B 229, 31 (2002).

[10] M. Godlewski, A. Wittlin, R.R. Gałązka, B. Monemar, T. Gregorkiewicz, C.A.J. Ammerlaan, P.H.M. van Loosdrecht, J.A.A.J. Perenboom, in: The Physics of Semiconductors, Eds. M. Scheffler, R. Zimmermann, in: Proc. XXIII Intern. Conf. ICPS'1996, Berlin 1996, Vol. 1, World Scientific, Singapore 1996, p. 393. 